\title{
Versatility of Anterolateral Thigh Flap in Dorsal Hand Reconstruction
}

\author{
MOUSTAFA MEKY, M.D. \\ The Department of Plastic and Reconstructive Surgery, Faculty of Medicine, Al-Azhar University, Cairo, Egypt
}

\begin{abstract}
Background: The latest advancement and high success rate of microsurgery cannot be denied. Although, free flaps have a pioneering characteristics among the rest of other options described in literatures for dorsal hand reconstruction; it should be emphasized that Selection of the donor site is very important. Anterolateral thigh flap was used in of head and neck, breast and upper and lower extremities reconstruction $[\mathbf{1 , 2}]$. In this article the application of this flap for the dorsal hand skin coverage is presented.
\end{abstract}

Material and Methods: Over almost 3 years (June 2013September 2016), 12 cases that required dorsal hand coverage were reconstructed by ALT flap. There were 11 males and 1 female with age range 8-49 years. Nine cases were post taumatic, and 3 cases were post burn. In all cases dorsal contractures release and adhesiolysis was carried out. Hand defect includes only the dorsum in 8 cases, the dorsum and $1^{\text {st }}$ web space in 1 case, and the dorsum of hand and fingers in 3 cases. The size of flap skin paddle ranged from $5-9 \mathrm{~cm}$ : $6-14 \mathrm{~cm}$ (Width: Length) ratio and the number of perforators included in each flap was single perforator in 10 cases and double in 2 cases. There was intercalary extensor tendon loss in 5 cases for them tensor fascia lata was used tendon reconstruction. There was tendon adhesions in 4 cases for them fascia lata was used to wrap around the tendons to prevent $r$ adhesions. In 1 case, there was metacarpal loss that was treated by ALT osteocutaneous flap. All flaps donor site was directly closed.

Results: Out of the 12 flaps harvested, only 1 flap failed completely secondary to unsalvageable vein thrombosis. Another 2 flaps developed partial flap necrosis, 1 of them left to heal by secondary intention and the other 1 required skin grafting. Flap debulking was required in 2 cases, 1 case in the stage of flap harvest, and the other case in a secondary stage. For TFL tendon reconstructed, they ended up with a very bad tendon excursion and even non functioning tendons. In the cases of tensor fascia lata wrapping an excellent tendon gliding was achieved. In ALT osteocutaneous flap metacarpal reconstructed case, sound bone healing and integration was achieved.

Conclusion: Purportedly, the author found in the robust characteristics of the ALT flap a warrant to be one of the best armaments in the reconstructive options of the hand dorsum.

Key Words: ALT-Hand-Dorsum - Reconstruction.

\section{INTRODUCTION}

Dorsum of the hand is the most visible part of the human body after the face. The skin is non glabrous, hirsute, thin and mobile to allow individual movements of joints. The very thin loose areolar layer underneath the skin and overlying the tendons acts as bolster that provides pliability and allows easy tendon gliding. When this skin is lost by any means, the ideal substitute should have the same character; thin, supple, and permits easy joint motion and tendon gliding [3].

Being thin and closely related to the underlying tendons, bone and joints, it is frequently uncommon to find isolated injury to the dorsal hand skin without affecting such structures [4]. Providing coverage should appreciate restoring of these structures, which can be carried out in the primary stage or in a protracted secondary or tertiary stages.

Although there are many donor sites for reconstruction of dorsal hand skin defects, reviewing literature revealed that free flaps demonstrate a high versatility especially in traumatic hand defects [5]. Free flaps can be harvested in almost any size, bringing its angiogenic and lymphogenic potentials [6], can improve arterial and venous flow in the already traumatized area by application of the flowthrough technique and can be made composite to provide additional vascularized tissue components like tendons, [7] bone, [8] and nerves $[\mathbf{9 , 1 0 ]}$ to the hand in addition to cover. It allows early hand elevation and physical therapy as it is usually a single stage procedure. The dorsalis pedis, [7] lateral arm, [11] scapular [8], TRAM [12], and temporoparietal fascia [13] flaps are among other free flaps that are used extensively in the reconstruction of this area of the body. These flaps demonstrate various versatilities and drawbacks.

Anterolateral thigh (ALT) flap nowdays is the standard flap for soft tissue reconstruction in dif- 
ferent areas of the body including head and neck, upper and lower extremity reconstruction $[\mathbf{1 , 1 4 , 2 1 , 2 2}]$ and has some indications in breast reconstruction [2]. This study demostarets the versatility of ALT for dorsal hand reconstruction.

\section{MATERIAL AND METHODS}

During the period from June 20113 till September 2016, 12 patients with dorsal hand trauma were reconstructed using ALT flap. There were 11 males $(91.6 \%)$ and 1 female $(8.4 \%)$. Their age ranged from 8-49 year (average 34.6 Y). Nine cases (75\%) resulting from industrial and road traffic accidents, and 3 cases $(25 \%)$ were due to burn trauma. All cases were treated on a secondary based schedule for treatment of dorsal contractures and adhesions following previous reconstructions by other means.

Hand defect was limited to hand dorsum in 8 cases, including the dorsum and palm in 1 case, and include the dorsum of hand and dorsal surface of fingers in 3 cases. The size of the flap skin paddle ranged from $5-9 \mathrm{~cm}: 6-14 \mathrm{~cm}$ (Width: Length) ratio. The number of perforators included in each flap was single in most cases (10 cases) and double perforator in 2 cases. Also the number of musculocutaneous perforators nourishing the flap was higher in comparison to the number of septocutaneous perforators ( $9: 3$ ratio). The mean pedicle length ranged from $7-12 \mathrm{~cm}$ (average $8.9 \mathrm{~cm}$ ).

In 5 cases there was intercalary extensor tendon loss ( 3 cases post traumatic, and 2 cases with post burn contracture). In these cases, vascularized tensor fascia lata was included in the flap and divided into multiple sleeves to reconstruct the lost extensor tendons. In 4 cases ( 3 post traumatic and 1 post burn), there was tendon adhesions, tenolysis was done in all but in 3 cases tensor fascia lata extention was used to wrapp around the tendons to prevent re-adhesions (Fig. 2B,C). In the case of metacarpal loss; ALT osteocutaneous flap was harvested and used for bone reconstruction and soft tissue coverage (Fig. 1A). All flaps donor site was closed directly without need for skin grafting.

\section{RESULTS}

Out of the 12 flaps harvested, only 1 flap related to post burn reconstructed cases failed completely secondary to vein thrombosis that was explored and revised but without success. Also another 2 flaps ( 1 for traumatic and 1 for post burn reconsted cases) developed partial flap necrosis, 1 left to heal by secondary intention and the other 1 required skin grafting. Donor site morbidity in the form of wound dehiscence was developed in 1 case and the rest cases healed completely. Regarding the flap thickness it was reasonable in 10 cases, and required debulking in 2 cases. Debulking was done in 1 case in the stage of flap harvest, and in a secondary stage in another case. In the 5 cases where TFL was used for tendon reconstruction, they developed very bad tendon excursion and even non functioning reconstructed extensor tendons. In the 4 cases of tensor fascia lata wrapping an excellent tendon gliding was achieved. In the case of ALT osteocutaneous flap used for reconstruction of metacarpal defects, sound bone healing achieved (Fig. 1B). In the 3 cases of surgical syndactalization, they were separated 2-4 months after primary surgery and they healed very well.
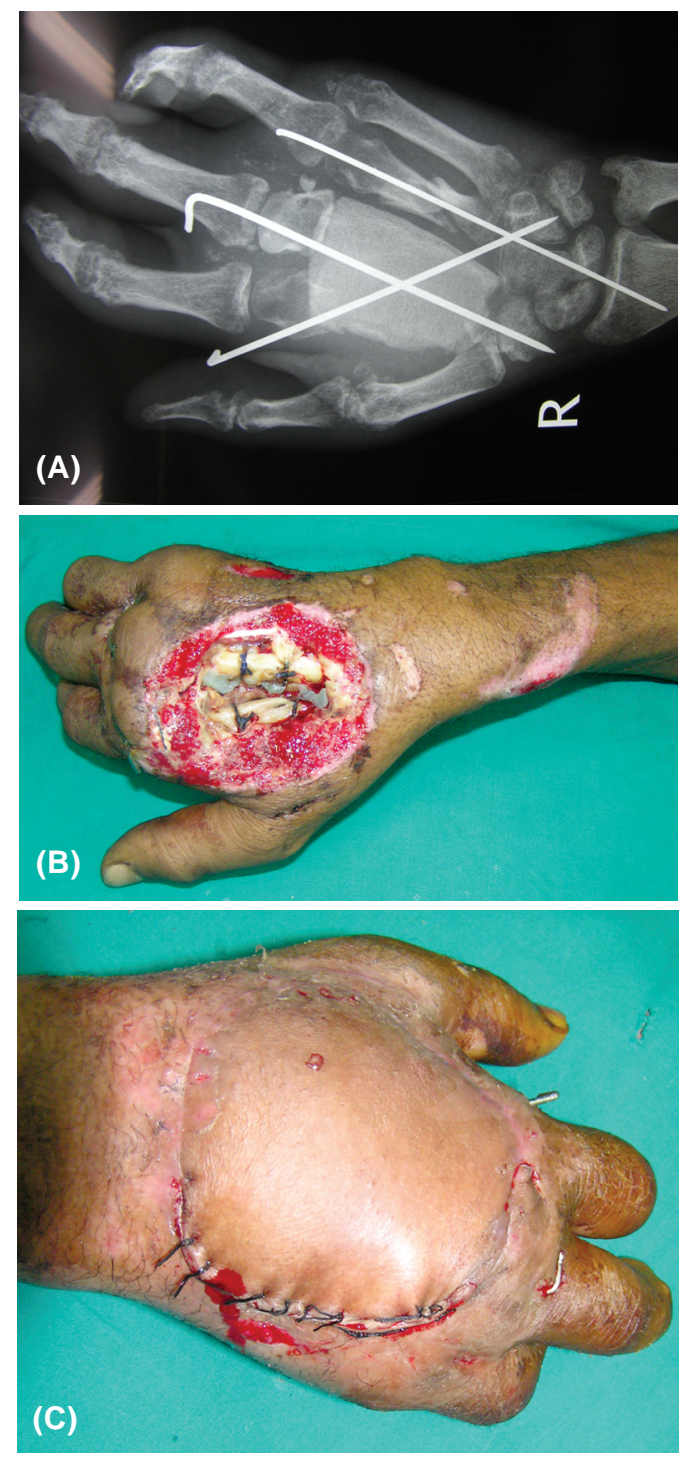

Case (1): Fig. (A) male patient 45 years old with complex dorsal hand defect including skin, tendons, and central metacarpal bone comminuted fractures with necrotic bone segments. Fig. (B,C) showing one month post operative photos following insertion of free ALT osteocutaneous flap to by pass the intercalary bone defect and skin cover. 

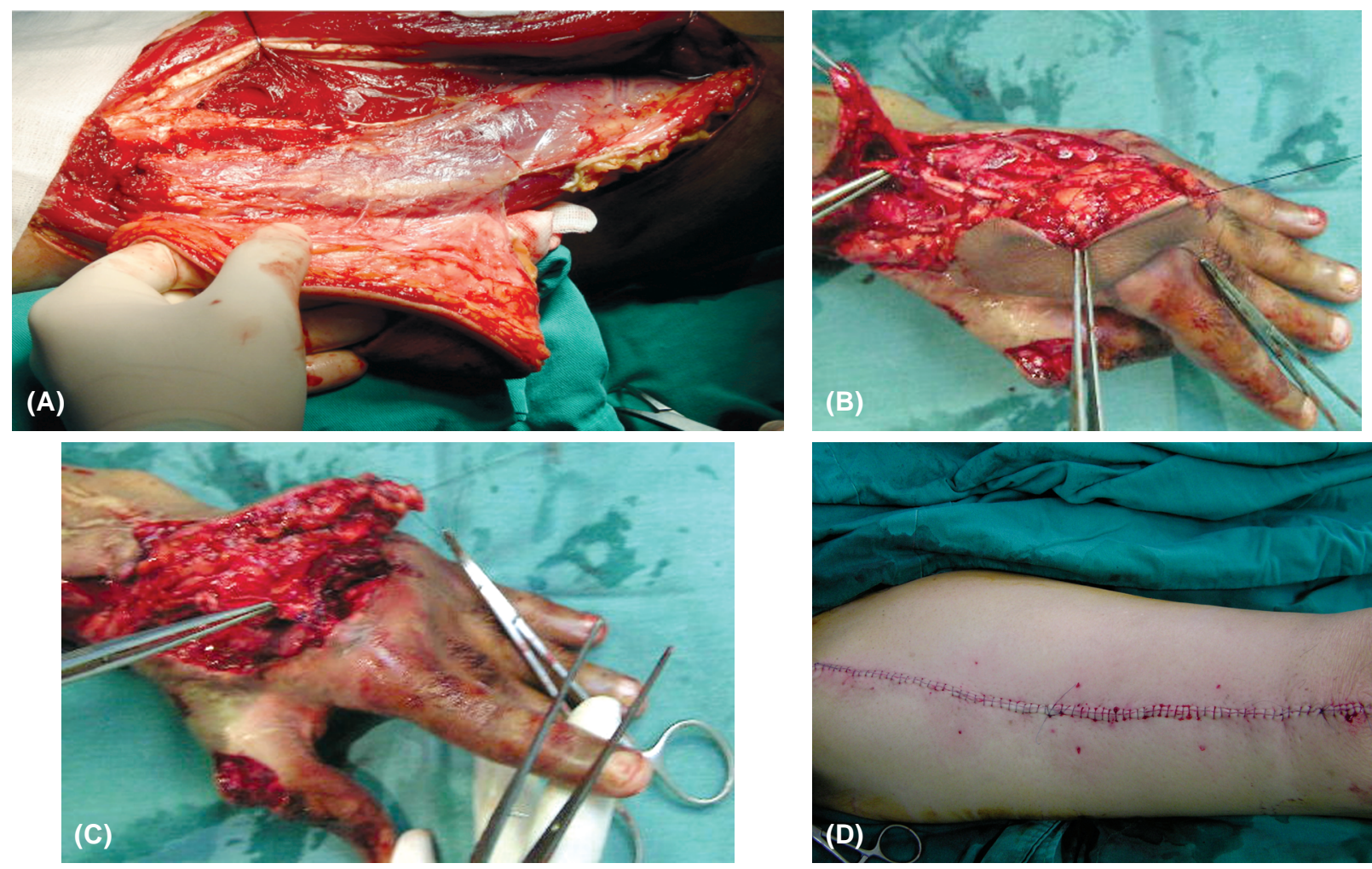

Case (2): Male patient 24 years old with old post traumatic adherent skin graft over previously repaired extensor tendons. Fig. $(A, B, C)$ shows harvesting of free ALT flap with vascularized fascia lata that was used to wrap the tendonlysed extensor tendons to provide gliding surface and prevent re-adhesions. Fig. (D) showing direct closure of the donor site of ALT flap without tension.
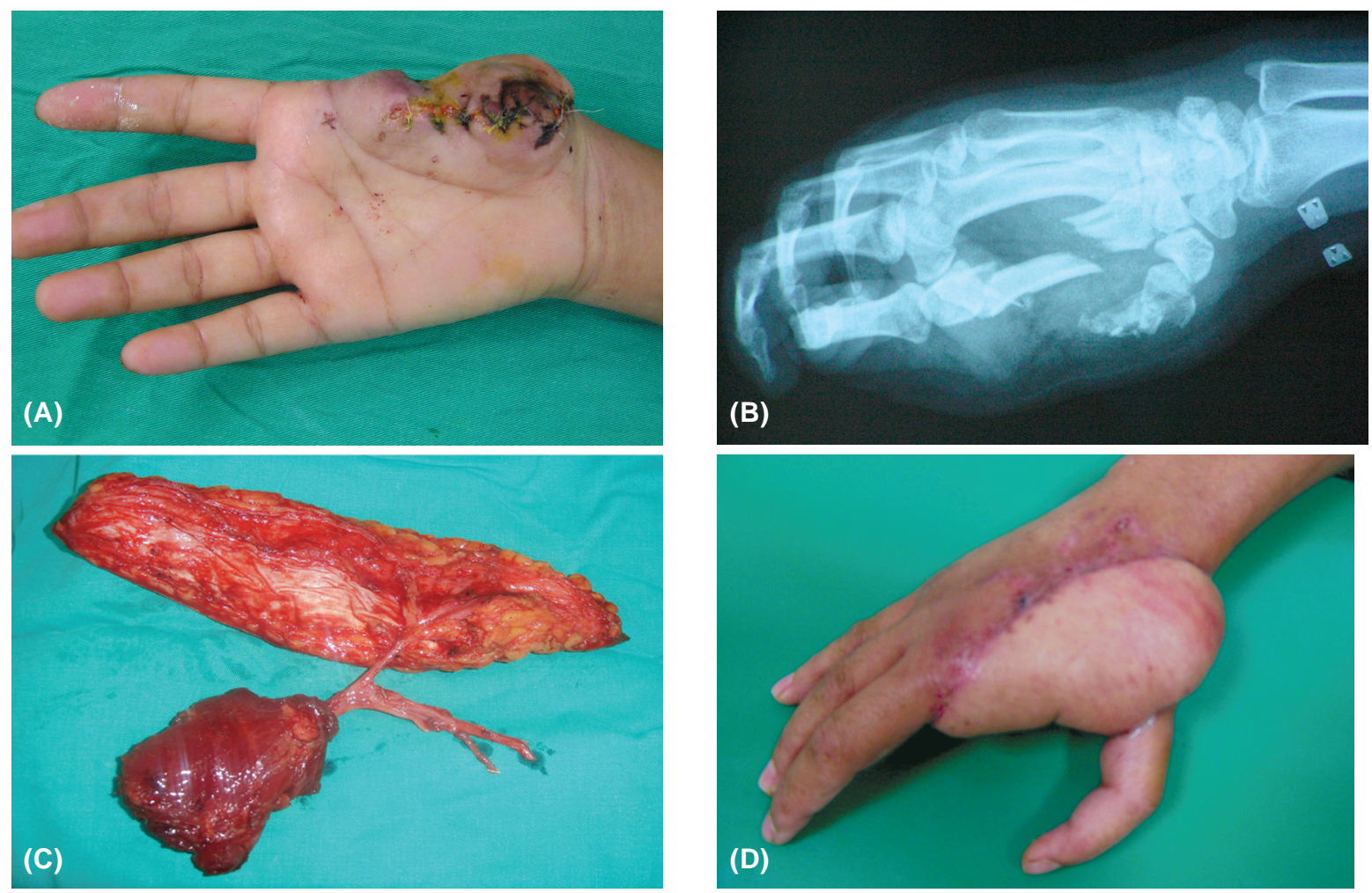

Case (3): Fig 3 (A,B) showing female patient 33 years old presented with post traumatic thumb amputation with fracture index at metacarpal base as shown from X-ray one month before. In this case policization of the fractured index finger was decided. Fig. (3C) showing harvested free chimeric ALT fasciocutaneous flap that include partial Vastus Lateralis muscle. The harvested muscle was used to obliterate the induced web space between the policized index and middle fingers. Fig. (3D) shows the result 2 months after surgery with viable flap and widened web space. 


\section{DISCUSSION}

Soft tissue defects over the dorsum of hand require scrutinized assessment and prudent surgical planning in order to restore optimal function and appearance. The nature of the skin in this area is of special surgical interest. It is thin and pliable for smooth tendon gliding. It is easily injured by trauma, which is frequently associated with extensor tendons and bone injuries $[\mathbf{4 , 2 1 , 2 2 ]}$. Isolated skin injury is straightforward, however, any skin replacement therapy could be thin. The thin characteristics of the ALT flap render it a suitable tool for dorsal hand coverage. Even with local adiposity, it is possible to thin the flap by removing excess fat underneath the skin up to the dermis [15]. The flap provides a pliable gliding surface for the extensor tendons and its long and large sized pedicle allows application of the flow-through pattern to improve vascularity in the injured hands. The hidden and permissible direct closure of its donor site adds superiority to this flap over the forearm, dorsalis pedis and lateral arm flaps. The use of ALT flap allows a tow-team approach with the patient in the supine position, and repositioning is not required which is the rule in the scapular flaps. Like groin flap, an ample skin paddle can be harvested; thigh flap surpasses the defeat of groin flap as early physiotherapy and elevation of the oedametous limb can be supervened.

When both skin and osseotendinous framework are injured, their reconstruction is carried out either through multistage surgical interventions the first rung of which is the primary skin cover, followed or combined with intercalary tendons and bone grafting $[\mathbf{7 , 1 6}$ or by composite flaps that contains more than one tissue plane in its structure. If tendon and/or bone grafts are considered, the soft tissue cover should be pliable enough to allow such tendon grafts to slide very well, prevent adhesion with bone and should allow secondary procedures like tenolysis. For this purpose, surgeons [13] advocated the temporoparietal fascial flap to wrap the tendons and even they tunneled the grafts inbetween the superficial and deep temporal fascial layers. This flap requires secondary skin grafting, which is inferior to skin flaps. Also there is no warrant to harvest flap from the head to provide pliable tissue to the dorsum of the hand inspite of plenty of donor sites that can afford such tissues and their harvest is accepted by the patient. Inclusion of a wide fascial extension beyond the limits of the skin paddle of the harvested thigh flap is valid [22] and can be used to wrap the tendon grafts. Such maneuver was applied in 2 cases in this series and good results were obtained. Although, com- posite flaps in different combinations (Tendinocutaneous [7,11], osseocutaneous [8.22], and neurosensory [10] is quite appealing, and have been described to reconstruct lost structures in a single stage procedure, however, the author's experience is that the best indications for such compound flaps in dorsal hand reconstruction is not much beneficial. ALT flap can be made sensate [25] by anastomosing the lateral femoral cutaneous nerve to one of the cutaneous nerves in the nearby forearm, can be made osteocutaneous by inclusion of part of hip bone [22], and can be made tendinocutaneous by fascia lata inclusion $[\mathbf{2 1 , 2 3 ]}$. In some cases, the author tried to reconstruct the extensor tendons based on the concept of compound "tendinocutaneous" flaps by including the tensor fascia latae in the flap and then split it into multiple sleeves that are sutured to both proximal and distal stumps of tendons, it was noticed in the follow-up period that such tendons do not function well. Also it was reported that the indication of vascularized osseocutaneous flaps is when bone loss exceeds $6-8 \mathrm{~cm}$ [4]. However, it is difficult to find metacarpal loss that exceeds this length. But this flap was used once to reconstruct the central metacarpal defects as a whole unit and it works well (Case 1). Donor site and flap related co-morbidities following use of the previously described compound flaps are well reported. In case of dorsalis pedis flap for example it is commonly associated with delayed healing of skin-grafted donor defect that results in ugly donor scar [7]. The lateral arm flap, with part of triceps tendon and/or humeral bone [11] carries unsightly visible donor scar for men and women a like. Also it is bulky in the hand and has a limited skin paddle; where up to $6-8 \mathrm{~cm}$ only can be harvested. The scapular osteocutaneous flap [8] with some of its advantages in hand reconstruction, is bulky and its harvest needs to transpose the patient during operation, which is undesirable in hand surgery. Although the previously counted comorbidites is much less in case of ALT flap, however its use through these combinations still needs more research and testing.

Lateral thigh flap has a large skin paddle with multiple perforators. It is safe to split the flap into multiple skin segments [24] and also to make it chimeric [1] that contains different pieces of skin and muscle each with at least one perforator with a common pedicle (Fig. 3C). These segments or pieces can be conformed to cover different areas of the hand. Also it is permissible to make a hole in the flap [17] to accommodate the transferred toe in case of toe-to hand transfer. ALT flap like other free flaps is not parasitic on the tissues for them they are transferred. Moreover, it has a long pedicle 
with wide vessel diameter, which can be used for reconstruction of lost arterial segment thus; improve hand vascularity based on the flow-through concept [1]. Its scar donor site is secluded and its harvest allows two-team approach with the patient in the supine position. This flap surpasses other donors in this issue, which is of great benefits especially when operating on multiple traumatized patients, where other surgical interventions are required.

Where up to $8 \times 20 \mathrm{~cm}$ skin paddle can be closed directly, the disadvantage of this flap comes when skin grafting of the donor site is required. In this situation a slight contour defect is mostly encountered. For the anatomic variations and sometimes absence of perforators found by the author and in other reports $[\mathbf{1 8 , 1 9 ]}$ the thigh region in general is robust of many perforators to search and dissect. Using Doppler detector for preoperative mapping of perforators may renders their intraoperative allocation easier. Also based on free style flap harvest [20], it is easy to search for the perforators by dissecting under the skin and following them to their main domain. This flap contains multiple perforators and even if one perforator is inadvertently cut the flap is still viable and it is easy to look for other perforators.

\section{REFERENCES}

1- Wei F.C., Jain V., Celik N., Chen H.C., Chuang D. and Lin C.H.: Have we found an ideal soft tissue flap?An experience with 672 Anterolateral thigh flap. Plast. Reconstr. Surg., 109: 2219, 2002.

2- Wei F.C., Suominen S., Cheng M.H., Celik N. and Lai Y.: Anterolateral thigh flap for Post mastectomy Breast Reconstruction. Plast. Reconstr. Surg., 110: 82-87, 2002.

3- Wintsch K. and Helaly P.: Free flap of gliding tissue. J. Reconstr. Microsurg., 2: 143, 1986.

4- Wood M.B.: Composite free flaps to the hand. Hand Clinics, 13 (2): 231-238, 1997.

5- Chen H.C., Buchman M.T. and Wei F.C.: Free flaps for soft tissue coverage in the hand and fingers. Hand Clinics, 15 (4): 541-554, 1999.

6- Salvin S.A., Upton J., Kaplan W.D. and Van den Abbeele A.D.: An investigation of lymphatic function following free tissue transfer. Plast. Reconstr. Surg., 99: 730-43, 1997.

7- Caroli A., Adani R., Castagnetti C., Pancaldi G. and Squarzina P.B.: Dorsalis pedis flap with vascularized extensor tendon for dorsal hand reconstruction. Plast. Reconstr. Surg., 92: 1326-30, 1993.

8- Masaki F., Takihero D., Ryuuichi M. and Kumi M.: Late reconstruction of two total metacarpal bone defects using lengthening devices and a double-barrel osteocutaneous free parascapular flap. Plast. Reconstr. Surg., 106: 1026,2000
9- Kimata y., Uchiyama K. and Ebihara S.: Comparison of innervated and noninnervated free flaps in oral reconstruction. Plast. Reconstr. Surg., 104: 1307, 1999.

10- Halbert F.C. and Wei F.C.: Neurosensory free flaps; Digits and Hand. Hand Clinics, 13 (2): 251-261,1997.

11- Hou S.M. and Liu T.K.: Vascularized tendon graft using lateral arm flap. Acta. Orthop. Scan., 64: 373-376, 1993.

12- Rao V.K. and Baertsch, A.: Microvascular reconstruction of the upper extremity with the rectus abdominis muscle. Microsurgery, 15: 746, 1994.

13- Upton J., Rogers C. and Durham-Smith G.: Clinical application of free temporoparietal flaps in hand reconstruction. J. Hand Surg., 11: 475, 1986.

14- Chao W., Chen H.C., Jain V., Kilda M., Lin Y., Chen M.H., Lin S.H., Chen Y.C., Tsai F.C. and Wei F.C.: Reconstruction of Through-and-Through cheek defects involving the oral commissure, using chimeric flaps from the thigh lateral circumflex femoral system. Plast. Reconstr. Surg., 109: 433, 2002.

15- Naohiro K., and Kaneshige S.: Consideration of a Thin Flap as an Entity and Clinical Applications of the Thin Anterolateral Thigh Flap.Plast. Reconstr. Surg., 97 (5): 985, 1996.

16- Scheker L.R., Langley S.J. and Martin D.L.: Primary extensor tendon reconstruction in dorsal hand defects requiring free flaps. Br. J. Plast. Surg., 18 (B): 568, 1993.

17- Rui Y., Shou k., Zhang Q., Xu Q., Sun Z. and Xu L.: Combined free-tissue for primary reconstruction of radial part of the hand. Microsurgery Jan., 59-62, 2004.

18- Kimata Y., Uchiyama K., Ebihara S., Nakatsuka T. and Harri K.: Anatomic variations and technical problems of the anterolateral thigh flap: A report of 74 cases. Plast Reconstr Surg., 102: 1517, 1998.

19- Celik N., Wei F.C., Lin C.H., Cheng M.H., Chen H.C., Jeng S.F. and Kuo Y.R.: The technique and strategy in Anterolateral thigh perforator flap based on an analysis of 15 complete and partial failures in 439 cases. Plast. Reconstr. Surg., 109: 2211-218, 2002.

20- Mardini S., Tsai F.C. and Wei F.C.: The thigh as a model for free style flaps. Clin. Plastic. Surg., 30: 473-480, 2003.

21- Yazar S., Gideroglu K., Kilic B., et al.: Use of composite anterolateral thigh flap as double-vascularised layers for reconstruction of complex hand dorsum defect. J. Plast. Reconstr. Aesthet. Surg., 61: 1549-50, 2008.

22- M. Meky and Y. Safoury: Composite anterolateral thigh perforator flaps in the management of complex hand injuries. J. Hand Surg. Eur. Vol. May, 38 (4): 366-70, 2013.

23- Man-Yi Cui and Hui Shen.: Anterolateral thigh free flap for simultaneous reconstruction of digital extensor tendon and defect of the dorsal hand: A case report. Chin. J. Traumatol. Oct., 13: 19 (5): 309-310, 2016.

24- Efficient design of split anterolateral thigh flap in extremity reconstruction. Plast. Reconstr. Surg. Dec., 128 (6): 12429, 2011.

25- Sensate anterolateral thigh perforator flap for ischiatic sores reconstruction in meningomyelocele patients. Microsurgery. May, 35 (4): 279-83, 2015. 